\title{
COMBINED BRAKING PERFORMANCE OF SHAPE MEMORY ALLOY AND MAGNETORHEOLOGICAL FLUID
}

\author{
Yang Xiong, Jin Huang, Rui Zhi Shu \\ College of Mechanical Engineering, Chongqing University of Technology, Chongqing, China \\ e-mail: xiongyang2019@qq.com; jhuangcq@cqut.edu.cn; ruizhishu@cqut.edu.cn
}

\begin{abstract}
To overcome the disadvantage of decreasing the braking performance of a magnetorheological fluid at high temperatures, a method of combined braking of a shape memory alloy and a magnetorheological fluid is proposed in this paper. The braking torque does not decrease with increasing temperature. Based on the magnetorheological characteristics, the braking torque equation of the magnetorheological fluid is established. Based on the characteristics of the thermal effect, the brake torque equations generated by the shape memory alloy springs are established. This paper provides a basis for the design and manufacture of the shape memory alloy and magnetorheological fluid combined brake system through experiments and theoretical analysis.
\end{abstract}

Key words: shape memory alloy, magnetorheological fluid, temperature, combined braking torque

\section{Introduction}

Shape memory alloys (SMAs) are a new class of smart alloy materials with the shape memory effect and superelasticity (Kim et al., 2006). The shape memory effect and superelasticity of shape memory alloys are due to different crystal structures of the materials at two different temperatures. The shape memory effect originates from the thermoelastic martensite transformation. When SMA is heated above a certain temperature, it will begin to transform from martensite to austenite. The different morphologies of martensite and austenite will cause reversible changes in the shape of SMA in the macroscopic view (Otsuka and Ren, 2005). The basic application of these materials is very simple, deforming a material by applying an external force at low temperatures, and shrinking or restoring the material to its original shape when heated beyond a certain temperature by external or internal heating (Mohd et al., 2014).

Since the discovery of shape memory alloys in 1932, scholars in various countries have constructed constitutive models of different types of shape memory alloys. The mechanical and thermal properties of shape memory alloys were analyzed in combination with experiments. Shape memory alloy springs designed based on the shape memory effect and superelastic properties have broad application prospects in the industrial field. Aguiar et al. (2010) described the thermomechanical shear behavior of a shape memory alloy based on a one-dimensional constitutive model and studied the thermomechanical behavior of shape memory alloy helical springs through experiments under different thermal loads. Mirzaeifar et al. (2011) simplified the analysis of helical springs to pure torsion analysis of straight rods with a circular cross-section, obtained the torsion response of shape memory alloy rods during loading and unloading, and analyzed mechanical properties of the shape memory alloy helical springs by using a simplified torsion model. An et al. (2012) demonstrated the static model of the shape memory alloy driver, which accurately described the force-deflection relationship of the driver in the martensitic and austenitic states. 
A magnetorheological (MR) fluid is a solid-liquid two-phase smart material whose morphology and performance are constrained and controlled by the applied magnetic field. The excellent performance of an MR fluid is that under the action of an external magnetic field, the MR fluid completes reversible transformation from a liquid to semisolid or plasticity in milliseconds (Rabinow, 1948; Jacob, 1951). During the increase of magnetic flux density, the apparent viscosity of these fluids can exhibit several orders of magnitude changes, producing shear yield strength similar to that of solids, and can be continuously controlled by the applied magnetic field (De et al., 2011). At present, the MR fluid has been widely used in dampers (Muthalif et al., 2017), clutches and brakes (Hwang et al., 2019).

Due to the unique and excellent performance of MRF, MRF-based brakes have significant advantages over conventional brakes. Li and Du (2003), basing on the Bingham plastic model, designed and fabricated a new type of an MR brak. Their experiment showed that the braking force increased with the increase of magnetic field intensity or rotational speed. Huang et al. (2002) proposed a design method of a cylindrical MR brake and deduced the torque equation of the MR fluid transfer in the brake, which provided a theoretical basis for the design of a cylindrical MR brake. Wang et al. (2013) proposed to use a water-cooling method to assist heat dissipation to solve the problem that a high-power MR brake is heated, which leads to attenuation of MR fluid performance. Le-Duc et al. (2018) proposed a multi-objective optimization method combining NSGA-II and FEA to seek the optimal solution under the conditions of maximizing braking torque and minimizing quality. Wang and Bi (2020) validated the method of applying pressure to an MR fluid to improve torque capability of the MR brake and deduced in detail the torque estimation formula of the MR fluid brake in the squeeze-shear mode. An MRF is fast in response and easy to control, but due to the low shear yield stress of the MRF, the torque transferred by a conventional MRF drives is small and the performance of the MRF decreases significantly at high temperatures, which limits the application of the MRF.

The above is mainly a single MR fluid or SMAs characteristics, and application research, especially in the study of an MR fluid transmission device, mostly does not solve the temperature effect on the MR fluid. An MR brake generates lots of heat when braking. When temperature exceeds the working temperature of the MR fluid, the rheological performance of the MR fluid significantly declines or even fails. In an earlier research work, our team innovatively proposed a combined transmission method of the MRF and SMA, which solved the performance degradation of the MRF at high temperatures. In this paper, an MR brake based on the shape memory effect of SMAs is proposed to solve the problem that the performance of MR fluid significantly decreases under high temperature. The proposed MR brake uses an SMA spring to drive a friction block to generate braking torque, which can compensate the braking performance of the MRF at high temperature. And provides a theoretical basis for the design and fabrication of novel MRF transmission devices.

\section{Working principle of MR brake}

The structure of the combined brake device of SMAs and MR fluid is shown in Fig. 1. The MR brake is composed of (1) left end cover, (2) bearing, (3) input shaft, (4) base, (5) right end cover, (6) MR fluid, (7) copper sleeve, (8) SMA spring, (9) friction block and (10) excitation coil.

When the MR brake is not working, the shape memory alloy spring does not reach the austenite transformation temperature, and the friction piston driven by the shape memory alloy spring does not contact with the inner cylinder of the base. When the coil is not electrified, the magnetic particles in the MRF are in a free state in the base fluid, and the liquid viscosity under zero magnetic field can hardly produce the braking effect. 
When the MR brake starts to work, the coil starts to energize and generates a circular magnetic field. Under the action of the magnetic field, the magnetic particles in the MR fluid are arranged in a chain structure along the direction of the magnetic field. Depending on this chain structure, the yield stress of the MRF can be increased. There must be an energy loss in the braking process, and most of the energy loss is converted to heat energy, which causes the MR brake temperature to rise. When temperature of the MR brake reaches the austenitic transition temperature of the SMAs, the SMA spring starts to output pressure and displacement. The friction piston is driven by the SMA spring and rubs with the inner cylinder of the base to produce braking torque.

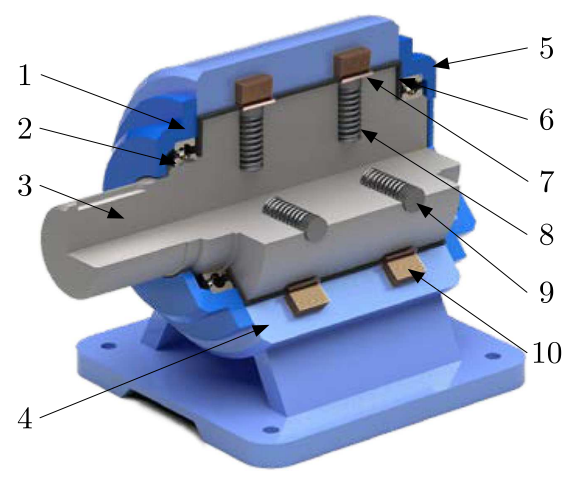

Fig. 1. Schematic diagram of a combined brake with SMAs and MR fluid

At the end of braking, the coil is disconnected from the power supply, the magnetic particles return to the free state in the base fluid, and the MR fluid does not produce the braking torque. As the brake temperature decreases, the SMA spring returns to its initial state, and the friction piston driven by the SMA spring is out of contact with the base inner cylinder.

\section{Braking torque equation}

\subsection{MR fluid torque characteristics}

\subsubsection{Cylindrical type of the MR fluid}

The cross section of the cylinder type of the MR fluid brake is shown in Fig. 2. Assume that the inner cylinder radius is $R_{1}$ and the radius of the outer cylinder is $R_{2}$. The MR fluid exists between the inner and outer cylinders, the angular velocity of the input shaft is $\omega_{1}$.

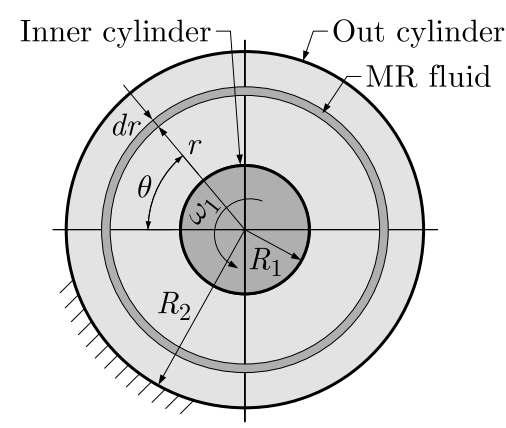

Fig. 2. Circumferential flow of the MR fluid between two cylinders

When the MR fluid is in circumferential shear flow, there is a micro-ring in the working gap of the MR fluid. In the cylindrical coordinate system $(r, \theta, z)$, take the length of the micro-ring along the $z$ axis direction as $d l$, projecting all forces generated by the MR fluid onto the 
tangential axis of the center of the micro-ring. Due to circumferential shear flow in the working gap, the tangential partial differential equation is

$$
\frac{1}{r} \frac{\partial \sigma_{\theta \theta}}{\partial \theta}+\frac{2 \tau_{r \theta}}{r}=0
$$

where $\sigma_{\theta \theta}$ is the tangential pressure along $\theta, \tau_{r \theta}$ is the shear stress of the MR fluid.

The constitutive model of the MR fluid with circumferential shear flow in the working gap is

$$
\begin{array}{ll}
\tau_{r \theta}=\tau_{y}(H)+\eta \dot{\gamma} & \tau_{r \theta} \geqslant \tau_{y}(H) \\
\dot{\gamma}=0 & \tau_{r \theta}<\tau_{y}(H)
\end{array}
$$

where $\tau_{y}(H)$ is the shear yield stress of the MR fluid under the magnetic field, $\eta$ is viscosity of the MR fluid, $\dot{\gamma}$ is the shear strain rate.

Partial differential equation (3.1) is solved as

$$
\tau_{r \theta}=\frac{c_{1}}{r^{2}}
$$

where $c_{1}$ is the integration constant.

When the radius of the working gap is $r$, and the rotating speed is $\omega_{r}$, the shear strain rate $\dot{\gamma}$ can be expressed as

$$
\dot{\gamma}=-\frac{r d \omega(r)}{d r}
$$

where $d \omega(r) / d r$ is the gradient of the angular velocity of the MR fluid flow along the radius direction.

Considering equations (3.2), (3.3) and (3.4), the rotational speed of the MR fluid at the radius $r$ is

$$
\omega(r)=\frac{\tau_{y}(H)}{\eta} \ln r+\frac{c_{1}}{2 \eta r^{2}}+c_{2}
$$

where $c_{2}$ is the second integration constant of the partial differential equation.

The MR fluid behave as Newtonian fluids when there is no external magnetic field $\left(\tau_{y}(H)=\right.$ $0)$. According to the boundary conditions, two integration constants of partial differential equations can be found

$$
c_{1}=\frac{2 \eta R_{1}^{2} R_{2}^{2}}{R_{2}^{2}-R_{1}^{2}} \omega_{1} \quad c_{2}=-\frac{R_{1}^{2} \omega_{1}}{R_{2}^{2}-R_{1}^{2}}
$$

and the angular velocity $\omega(r)$ is

$$
\omega(r)=\frac{R_{1}^{2} R_{2}^{2}}{R_{2}^{2}-R_{1}^{2}} \frac{R_{2}^{2}-r^{2}}{R_{2}^{2} r^{2}} \omega_{1}
$$

When the speed of the input shaft is $\omega_{1}$, the working gap length of the MR fluid is $L$. Then the braking torque of the MR brake is

$$
M=2 \pi r^{2} L \tau_{r \theta}
$$

Considering equations (3.2), (3.4) and (3.7), equation (3.8) of the cylinder type MR brake can be written as

$$
\eta d \omega(r)=\left(\frac{M}{2 \pi r^{3} L}-\tau_{r \theta} r\right) d r
$$

By integrating both sides of equation (3.9), the torque produced by the cylindrical MR brake can be obtained as (Huang et al., 2002)

$$
M_{c}=\frac{4 \pi R_{1}^{2} R_{2}^{2}}{R_{2}^{2}-R_{1}^{2}}\left(\tau_{r \theta} L \ln \frac{R_{2}}{R_{1}}-\eta L_{e} \omega_{1}\right)
$$

where $L_{e}$ is the effective working gap length of the MR fluid. 


\subsubsection{Disc type of the $M R$ fluid}

The cross section of the disc type MR fluid brake is shown in Fig. 3. Assume that $R_{3}$ is the radius of the inner circle of the disc, $R_{4}$ is the radius of the outer circle of the disc, the magnetorheological fluid exists between the two discs, and the angular velocity of the input axis is $\omega_{1}$.

Because the electromagnetic field is a kind of a non-uniform magnetic field, the chaining of the MRF is also non-uniform. In order to simplify the complexity of the formula, it is assumed that the shear stress in the MRF gap is consistent. When the whole MR fluid is yielding to shear flow, the flow equation along the rotation direction of the micro-ring $\theta$ is

$$
\frac{d^{2} \omega(z)}{d z^{2}}=\frac{1}{\eta} \frac{d p}{d \theta}
$$

Under laminar boundary conditions, when $z=0, \omega(z)=\omega_{2}$. When $z=h, \omega(z)=\omega_{1}=0$. Integrating equation (3.11), the MR fluid flow equation is

$$
\omega(z)=\frac{\omega_{1} z}{h}+\frac{1}{2 \eta} \frac{d p}{d \theta}\left(z^{2}-z h\right)
$$

The shear stress produced by the MR fluid in the circumferential shear flow is shown in Eq. (3.2). The torque transmitted by the disc type MR brake is

$$
M_{d}=\int_{R_{3}}^{R_{4}} d M=2 \pi \int_{R_{3}}^{R_{4}} \tau r^{2} d r
$$

Considering equations (3.2), (3.12) and (3.13), the torque of the disc type MR brake is

$$
M_{d}=\int_{R_{3}}^{R_{4}} 2 \pi \tau r^{2} d r=\frac{2 \pi}{3}\left(R_{4}^{3}-R_{3}^{3}\right) \tau_{y}(H)+\frac{\pi}{2 h}\left(R_{4}^{4}-R_{3}^{4}\right) \omega_{1} \eta
$$

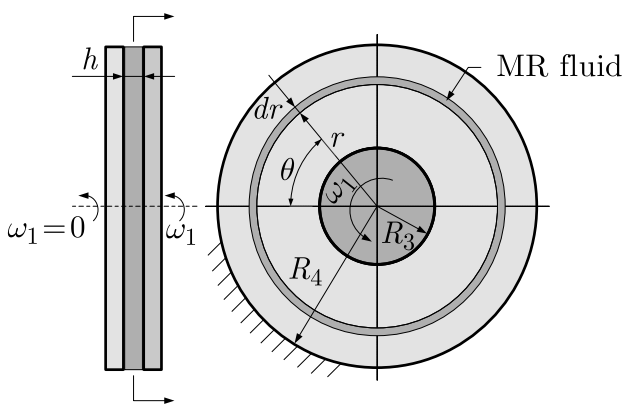

Fig. 3. Circumferential flow of the MR fluid between two discs

\subsubsection{MR fluid performance at high temperature}

At high temperature, the shear stress, viscosity and rheological characteristics of the MR brake are significantly reduced, and the damage of thermal stress to the structure of the MRF chain changes, which makes the performance of the brake attenuate significantly at high temperature. Sun et al. (2013) observed that the shear yield strength of an MRF (composition: silicone oil, carbonyl iron particles; density $3.6 \mathrm{~g} / \mathrm{cm}^{3}$; viscosity: $0.28 \mathrm{~Pa}$ s; solids content by weight: 


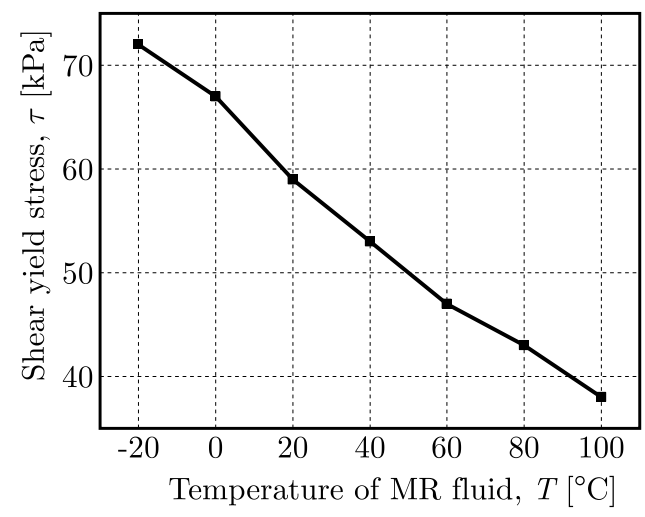

Fig. 4. Shear yield stress of the MRF at different temperatures

$85.44 \%$ ) decreased linearly when temperature increased. Their experimental results are shown in Fig. 4. Using the least square method, the fitting equation is

$$
\tau=-0.29 T+66
$$

where $\tau$ is the shear yield stress of the MR fluid, $T$ is temperature of the MR fluid.

In order to describe the relationship between magnetic flux density and shear yield stress of the MR fluid at different temperatures, based on experimental data. The shear yield stress of the MR fluid at high temperatures is

$$
\tau(B)_{T_{i+n}}=n \mu_{d} \tau(B)_{T_{i}}
$$

where $\mu_{d}$ is the attenuation coefficient of the shear yield stress of the MR fluid at high temperature. And the attenuation coefficient $\mu_{d}$ can be expressed as

$$
\mu_{d}=\frac{1}{n-1} \sum_{i=1}^{n} \frac{\tau_{i+1}}{\tau_{i}}=\frac{1}{n-1} \sum_{i=1}^{n} \frac{-0.29 T_{i+1}+66}{-0.29 T_{i}+66}
$$

The relationship between the magnetic flux density of the MRF and the shear yield stress at different temperatures is obtained by Eq. (3.16), as shown in Fig. 5.

(a)

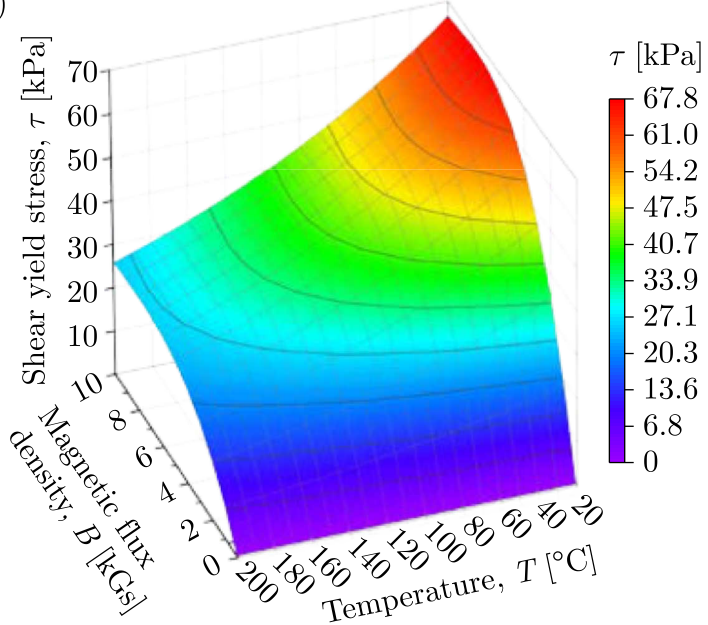

(b)

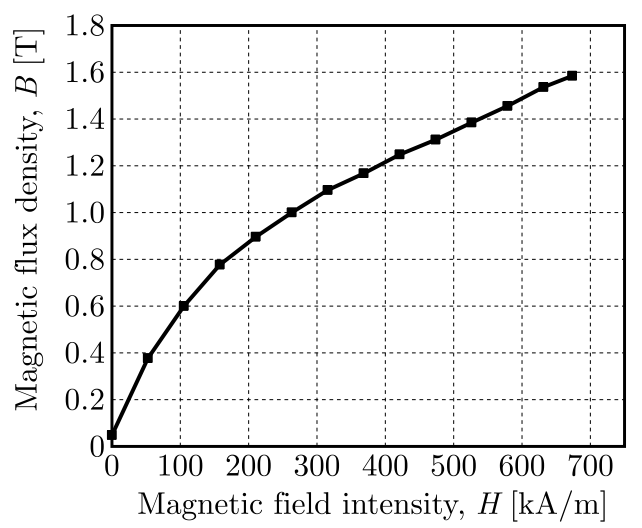

Fig. 5. (a) Relationship between the magnetic induction intensity and the shear yield stress at different temperatures; MR fluid magnetization curves

When the MR fluid temperature is $20^{\circ}, 40^{\circ}, 60^{\circ}, 80^{\circ}, 100^{\circ}$, the maximum shear yield stresses are $68.21 \mathrm{kPa}, 61.20 \mathrm{kPa}, 54.43 \mathrm{kPa}, 46.61 \mathrm{kPa}$ and $43.82 \mathrm{kPa}$, respectively. When the MR fluid 
temperature rises from $20^{\circ}$, to $100^{\circ}$, the maximum shear yield stress decreases by $36.76 \%$. It can be seen from equations (3.10) and (3.14) that the temperature of the MR fluid has a linear relationship with the shear stress and braking torque.

\subsection{SMA spring torque characteristics}

The thermal energy generated by the MR brake coil and the MR fluid slip is used to drive the SMA spring to output friction torque, thus compensating for the performance attenuation of the MR fluid at high temperature.

If the deformation of the spring is restrained during the phase transition of the shape memory alloy, the spring generates an extrusion force $F_{e}$ on the restraint body. The extrusion force of the SMA is related to ambient temperature and constrained deformation. When the temperature of the shape memory alloy is between the martensitic transition temperature $M_{f}$ and the austenitic transition temperature $A_{f}$, the spring extrusion force is (Ma et al., 2013)

$$
F_{e}=\frac{\delta(H) G(H)}{\delta_{L} G_{L}} F_{L}
$$

where $\delta(T)$ is the axial expansion of the spring, $G(T)$ is the shear modulus of the SMA, $\delta_{L}$ is the axial expansion at temperatures below the austenitic transition temperature $A_{s}, G_{L}$ is the shear modulus of the SMA at temperatures below $A_{s}$.

The axial load of the SMA spring at low temperature is

$$
F_{L}=\frac{d^{4} G_{L}}{8 D^{3} n} \delta_{L}
$$

where $d$ is the wire diameter, $D$ is the spring mid-diameter, $n$ is the number of effective spring bearing cycles.

When restraining the axial displacement of the SMA spring, the axial expansion of the spring remains constant $\delta(H)=\delta_{L}$. Considering equations (3.18) and (3.19), when the temperature of the shape memory alloy is between $M_{f}$ and $A_{f}$, the spring extrusion force $F_{r}$ is

$$
F_{e}=\frac{G(H) d^{4}}{8 D^{3} n} \delta_{L}
$$

The friction torque produced by the SMA spring by applying the extrusion force $F_{r}$ to the inner wall of the MR brake base is

$$
M_{s}=N \mu F_{e} R_{2}=N \mu \frac{G(H) d^{4}}{8 D^{3} n} \delta_{L} R_{2}
$$

where $N$ is the number of SMA springs, $\mu$ is the coefficient of friction between the frictional block and the copper sleeve.

\subsection{Combined braking torque}

The combined braking torque is obtained by superposing the braking torque generated by the MR fluid of the disc working gap (left and right discs formed by the cylinder end face and the shell wall), the braking torque generated by the MR fluid of the cylinder working gap and the braking torque generated by the SMA spring. The combined braking torque $M$ is

$$
\begin{aligned}
M= & 2 M_{d}+M_{c}+M_{s}=\frac{4 \pi}{3}\left(R_{4}^{3}-R_{3}^{3}\right) \tau_{y}(H)+\frac{\pi}{h}\left(R_{4}^{4}-R_{3}^{4}\right) \omega_{1} \eta \\
& +\frac{4 \pi R_{1}^{2} R_{2}^{2}}{R_{2}^{2}-R_{1}^{2}}\left(\tau_{r \theta} L \ln \frac{R_{2}}{R_{1}}-\eta L_{e} \omega_{1}\right)+N \mu \frac{G(H) d^{4}}{8 D^{3} n} \delta_{L} R_{2}
\end{aligned}
$$




\section{Experimental setup}

\subsection{Extrusion force test of the SMA spring}

The schematic diagram of the SMA spring extrusion force experimental system is shown in Fig. 6a. The test system can test the extrusion force of the SMA spring under different temperatures when the axial displacement is restrained. To reduce the temperature drop caused by the heat exchange between the device and the external environment, the SMA spring needs to be heated by a thermostat. Slow heating of SMA springs is required in order to allow adequate heat exchange and to reduce uneven heating. In the experimental system, the DC power supply provides current to the electric heating wire in the thermotank. The SMA spring is placed on a fixed base with the top in contact with the dynamometer but without a force. A temperature sensor is attached to the surface of the SMA spring. The current sensor measures the current through the electric heating wire. The data measured by the dynamometer, temperature sensor and current sensor are transmitted to the computer through an A/D card.

(a)

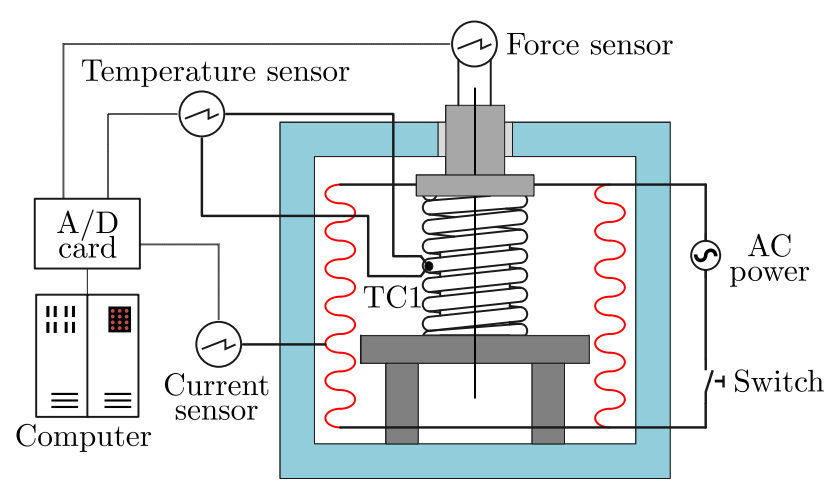

(c)

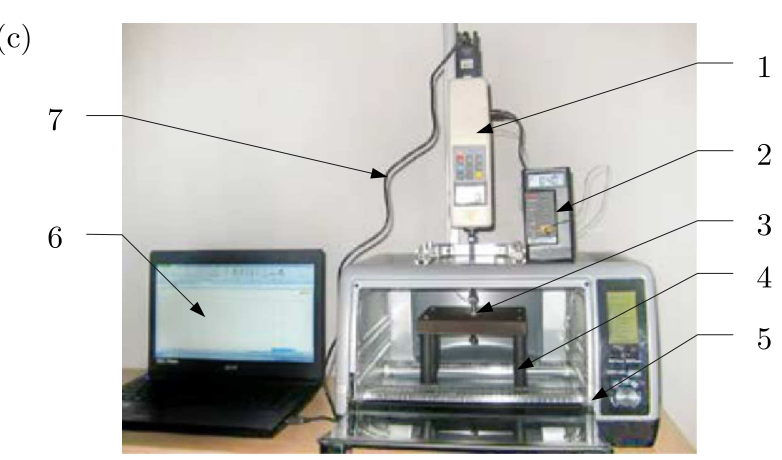

(b)

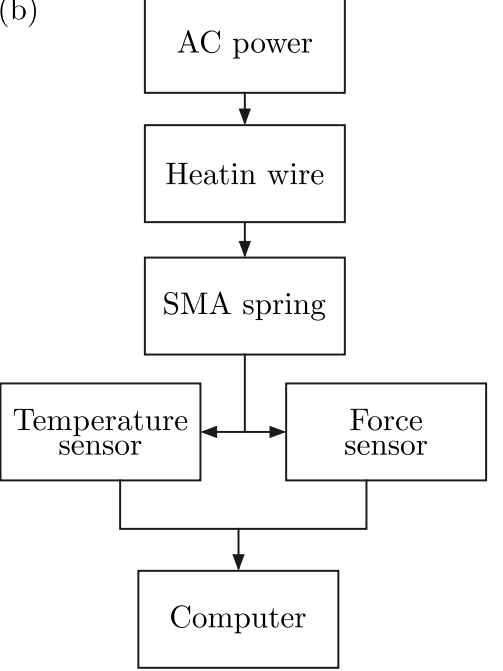

Fig. 6. (a) Schematic diagram of the SMA spring extrusion force experiment; (b) experimental flow chart; (c) experimental system for measuring the extrusion force of the SMA spring

The experimental system for measuring the relationship between the extrusion force and temperature of the SMA spring is shown in Fig. 6b. The devices mainly involved in the experiment include: (1) SH-200N digital dynamometer (maximum measuring range $200 \mathrm{~N}$, resolution $0.1 \mathrm{~N}$ ), (2) TES-1310 digital thermometer (measuring range is $-50^{\circ}$, to $200^{\circ}$, resolution $0.1^{\circ}$ ), (3) SMA spring. The structural parameters are: spring pitch diameter $D=8.6 \mathrm{~mm}$, wire diameter $d=1 \mathrm{~mm}$, number of active coils $n=7$, spring inclination $\alpha=6^{\circ}$. The material parameters of SMAs are shown in Table 1. (4) is the SMA spring fixed base, (5) thermotank, (6) computer, (7) RS232 data transmission line. 
Table 1. Material properties of SMA

\begin{tabular}{|c|c|c|c|c|}
\hline$A_{s}\left[{ }^{\circ} \mathrm{C}\right]$ & $A_{p}\left[{ }^{\circ} \mathrm{C}\right]$ & $A_{f}\left[{ }^{\circ} \mathrm{C}\right]$ & $E_{a}[\mathrm{GPa}]$ & $C_{a}\left[\mathrm{MPa}^{\circ} \mathrm{C}\right]$ \\
\hline \hline 63.28 & 83.53 & 92.91 & 230.0 & 13.80 \\
\hline$M_{s}\left[{ }^{\circ} \mathrm{C}\right]$ & $M_{a}\left[{ }^{\circ} \mathrm{C}\right]$ & $M_{f}\left[{ }^{\circ} \mathrm{C}\right]$ & $E_{m}[\mathrm{GPa}]$ & $C_{M}\left[\mathrm{MPa}^{\circ} \mathrm{C}\right]$ \\
\hline \hline 49.47 & 43.81 & 36.21 & 67.0 & 8.0 \\
\hline
\end{tabular}

The SMA spring extrusion force test process was as follows. At first, connection between the bottom of the shape memory alloy spring and the base of the experimental device was fixed. In the second step, the dynamometer was adjusted to contact the top of the SMA spring without producing a force. In the third step, the dynamometer, temperature sensor and current sensor were turned on and used the multimeter to manually detect whether the connection of the experimental device was correct and whether each device works normally. In the fourth step, the computer was turned on to check whether the communication between various sensors and the computer was normal. In the fifth step, a small current was introduced into the heating wire in the thermotank, which caused the temperature of the whole thermostat to rise slowly. Finally, the measured data received in the computer ware exported and analyzed.

\subsection{Combined braking test}

The schematic diagram of the experimental system of combined braking of the SMA and MR fluid is shown in Fig. 7a. The experimental device was used to test the combined braking performance and to evaluate the accuracy of the theoretical design. In the experimental system, the motor, reducer, torque sensor and brake are interconnected by couplings (C1, C2, C3). The torque sensor is used to measure the braking torque. The Gauss gauge probe passes through a hole in the brake housing and is placed in the MR fluid working gap to detect the magnetic flux density. Similarly, the probe of the temperature sensor passes through the hole in the brake housing and is placed in the MRF working gap to detect temperature.

(a)

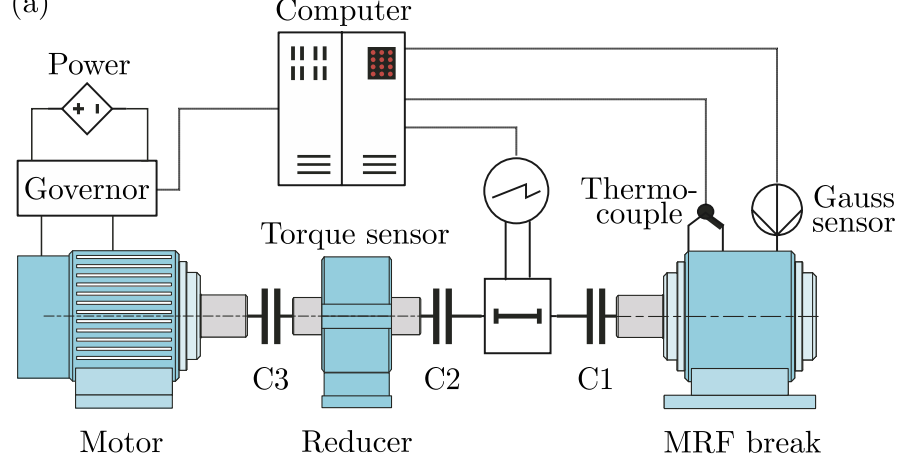

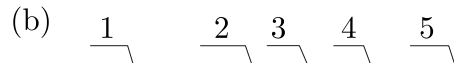

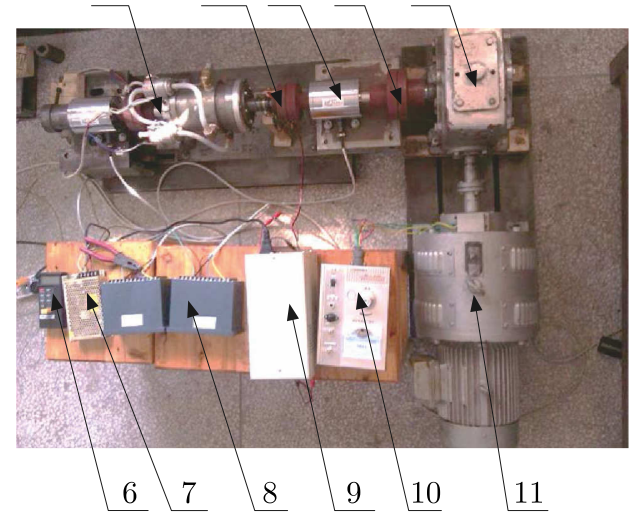

Fig. 7. (a) Schematic diagram of the combined braking experiment with the SMA and MR fluid;

(b) SMA and MRF combined braking experimental system

The experimental device for combined braking with the SMA and MR fluid is shown in Fig. 7b. The devices involved in the experiment include: (1) combined brake of the SMA and MR fluid, (2) coupling, (3) LDN-08D speed and torque sensor (maximum measuring range $200 \mathrm{Nm}$, resolution $0.1 \mathrm{Nm}$ ), (4) coupling, (5) WD100-1 worm gear reducer (transmission ratio 9.67), (6) TES-1310 digital thermometer (measuring range is $-50^{\circ}$ to $200^{\circ}$, resolution $0.1^{\circ}$ ), (7) DC power supply, (8) MCK-S dynamic torque digital display instrument, (9) RXN-3010D digital DC power supply (maximum output voltage $30 \mathrm{~V}$, maximum output current $10 \mathrm{~A}$, volt- 
age resolution $0.1 \mathrm{~V}$, current resolution 0.01 A), (10) JZT3 motor controller (excitation current 0.79 A), (11) YCT160-4B AC electro-magnetic speed-adjustable motors (rated torque $19.5 \mathrm{Nm}$, speed regulation range is from $120 \mathrm{rpm}$ to $1200 \mathrm{rpm}$, excitation voltage $90 \mathrm{~V}$ ).

To prevent precipitation of the MR fluid when placed in the brake for a long time, a new MR fluid was filled through the filling port of the brake at the beginning of the experiment. The combined braking test process is as follows. At first, the experimental equipment is installed in the designed location. The second step, the power supply of each sensor after connection of each sensor is completed. The third step, the motor is adjusted to speed of $600 \mathrm{rpm}$ through the motor controller. In the fourth step, after the motor reaches the specified speed, the torque sensor, Gauss gauge and thermometer begin to record the data. Finally, the data from the torque sensor, Gauss meter and temperature sensor are transmitted to the computer for export and analysis.

\section{Results and discussions}

\subsection{SMA spring extrusion force}

The experimentally measured and theoretically calculated extrusion forces of the SMA spring at different temperatures are shown in Fig. 8.

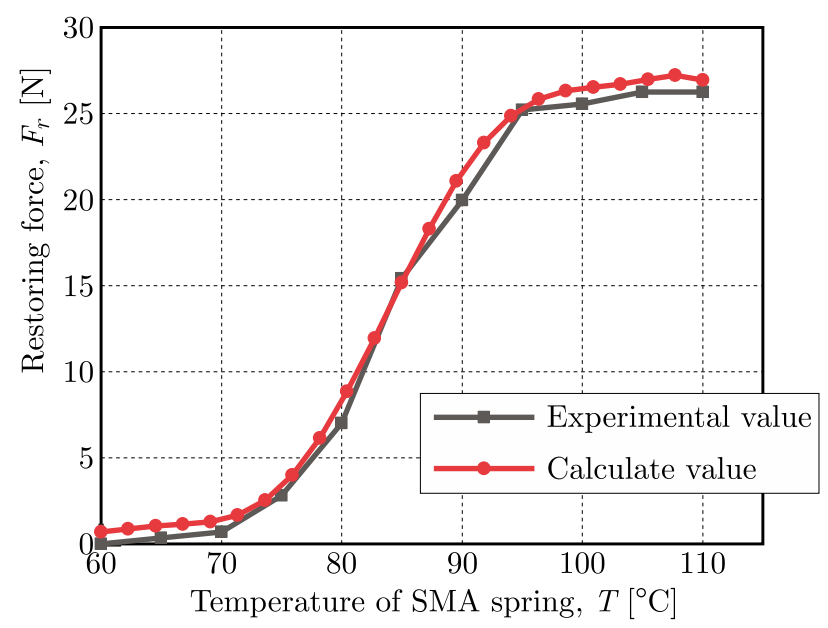

Fig. 8. Extrusion force of the SMA spring at different temperatures

As can be seen from Fig. 8, the extrusion force produced by the SMA spring can be divided into three stages under constant current heating. In the first stage, temperature of the SMA spring increases from $60^{\circ}$ to $73^{\circ}$, and the extrusion force increases slowly from $0 \mathrm{~N}$ to $2.5 \mathrm{~N}$. In the second stage, temperature of the SMA spring increases from $73^{\circ}$ to $94^{\circ}$, and the extrusion force increases rapidly from $2.5 \mathrm{~N}$ to $24.9 \mathrm{~N}$. In the first stage, temperature of the SMA spring increases from $93^{\circ}$ to $100^{\circ}$, and the extrusion force increases slowly from $24.9 \mathrm{~N}$ to $26.54 \mathrm{~N}$. Finally, when the SMA spring temperature exceeds $100^{\circ}$, the extrusion force produced does not increase any more.

The relative error between the theoretically calculated and experimentally measured extrusion force of the SMA spring at different temperatures is shown in Fig. 9. It can be seen from Fig. 9 that the theoretical calculated values are basically in agreement with the experimentally measured values. The error between the theoretically calculated and experimentally measured values is the greatest at $90^{\circ}$, and the relative error is $-7.9 \%$. The errors are mainly caused by fabrication of the SMA spring, system errors during experimental measurement and simplified treatment of the theoretical models. 


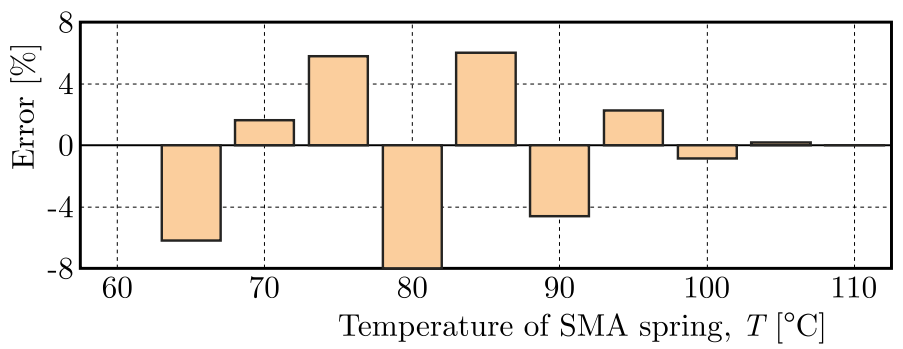

Fig. 9. The relative error between the values of calculation and experiment

\subsection{SMA braking torque}

The braking torque produced by the SMA spring at different temperatures is shown in Fig. 10.

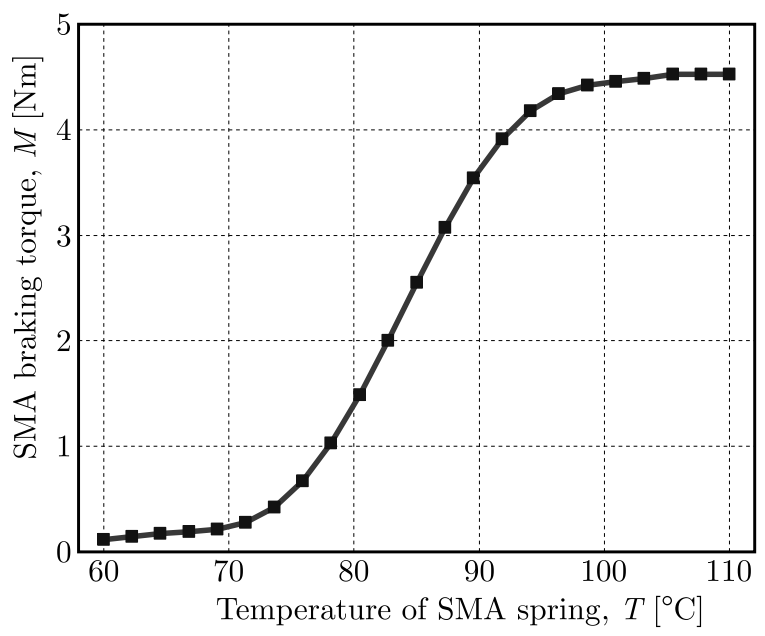

Fig. 10. SMA spring braking torque

As shown in Fig. 10, when temperature of the SMA is below $60^{\circ}$, no phase transformation occurs in the SMA, and the SMA spring does not produce braking torque. When temperature exceeds $60^{\circ}$, the inverse transformation of martensite from non-twin martensite to austenite occurs in the SMA. Depending on the shape memory effect, the SMA spring starts to output braking torque. When temperature of the SMA spring exceeds $100^{\circ}$, the braking torque will reach a maximum of $4.83 \mathrm{Nm}$. At this time, all non-twin martensite in the shape memory alloy will transform into austenite and, even if temperature continues to increase, the shape memory alloy spring will not continue to increase the braking torque.

\subsection{MRF braking torque}

The relationship between brake temperature and braking torque is shown in Fig. 11. The braking torque of the $\mathrm{MR}$ fluid is $10.1 \mathrm{Nm}$ at $20^{\circ}$, and decreases to $6.5 \mathrm{Nm}$ when temperature increases to $100^{\circ}$. The brake temperature rises due to coil heating and MR fluid slip heating. When the brake relies on a single MR fluid for braking, the braking performance of the MR fluid will gradually decrease as temperature rises.

\subsection{Combined braking torque}

The relationship between brake temperature and braking torque is shown in Fig. 12 . 


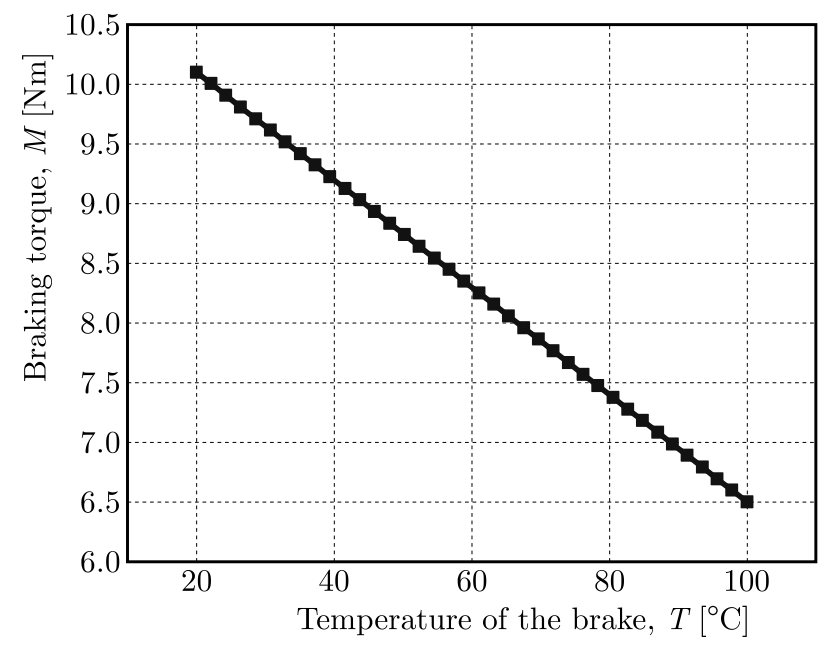

Fig. 11. MRF braking torque

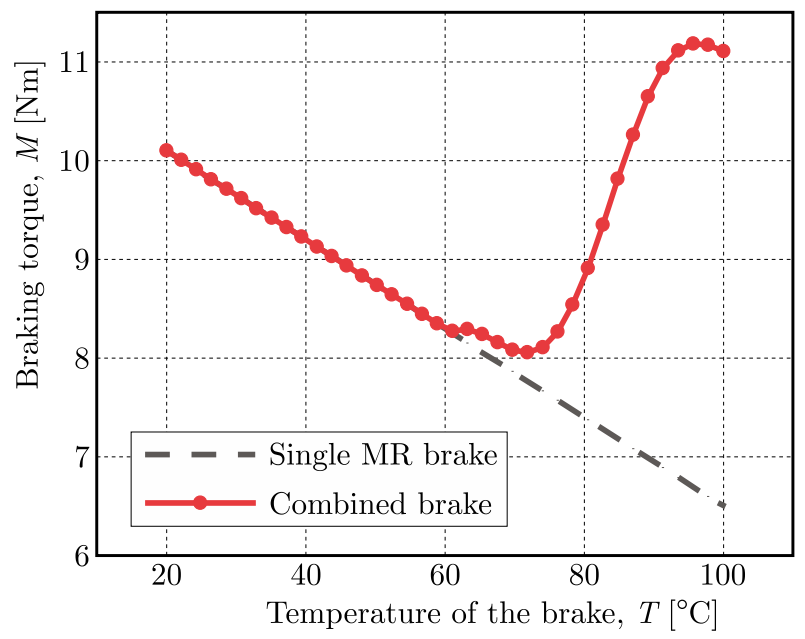

Fig. 12. Combined braking torque

From the combined braking torque curve in Fig. 12 it can be seen that the SMA spring starts to participate in braking when the brake temperature rises to $60^{\circ}$. When the brake temperature reaches $74^{\circ}$, the braking torque will not decrease any more. During the continuous increase of the brake temperature up to $100^{\circ}$, the braking torque continues to increase and reaches a maximum of $11.2 \mathrm{Nm}$. The maximum torque generated in this device at the room temperature is $10.2 \mathrm{Nm}$ (Ma, 2013). The effect of temperature rise on the transmission performance has not been considered in previous studies. The transmission performance of the MRF decreases linearly during the process of temperature rise, but the reduction of MRF performance at high temperature can be effectively compensated by the combined braking by the SMA and MRF, so that the transmission performance is stable.

The experimental and theoretical values of the braking torque at different temperatures are shown in Fig. 13.

It can be seen from Fig. 13 that the theoretical calculation value of the braking torque is basically consistent with the variation rule of the experimental measurement value. When the braking device temperature is between $60^{\circ}$ and $80^{\circ}$, the theoretical calculation value of the braking torque deviates greatly from the experimental measurement value. The reason why the calculation value is different from the experiment is that the errors caused by shear stress of the MR fluid are omitted and the magnetic leakage is neglected. 


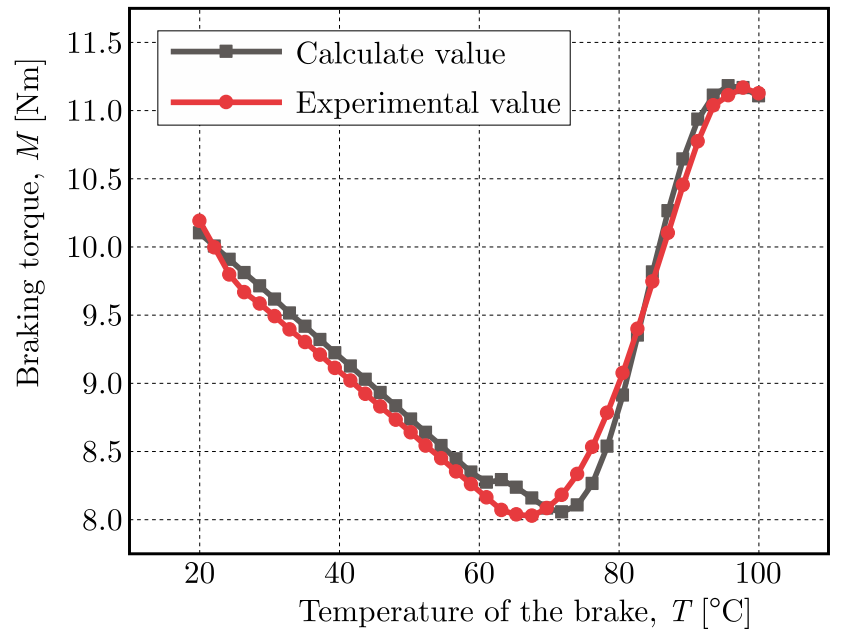

Fig. 13. Combined braking torque applied in calculations and the experiment

\section{Conclusions}

In this paper, a braking method combining an SMA spring and an MRF is proposed, and the structure and working principle of the braking device are elaborated. The shear stress of the $\mathrm{MRF}$ at different temperatures is calculated. The torque equations of the cylindrical and disc type braking devices are derived, the squeezing force of the SMA spring at different temperatures is calculated and, finally, the combined braking torque of the braking device at different temperatures is derived. The shear stress of the MRF at different temperatures is calculated. Torque equations for the cylindrical and disc braking devices have been derived. The squeezing force of the SMA spring at different temperatures is calculated. Finally, the combined braking torque of the braking device at different temperatures is determined.

Experiments have shown that the SMA spring starts to generate a squeezing force when temperature reaches $60^{\circ} \mathrm{C}$, and the squeezing force of the spring reaches the maximum of $26.5 \mathrm{~N}$ when the temperature rises to $100^{\circ} \mathrm{C}$. The maximum shear yield stress of the MRF decreases from $68.21 \mathrm{kPa}$ to $43.82 \mathrm{kPa}$ as temperature of the braking device increases from $20^{\circ} \mathrm{C}$ to $100^{\circ} \mathrm{C}$. The braking torque generated by the MRF gradually decreases as temperature of the braking device increases. When the MRF temperature rises to $100^{\circ} \mathrm{C}$, the maximum braking torque generated by a single MRF decreases down to $6.5 \mathrm{Nm}$, but the maximum torque generated by the combined braking with the SMA and MRF is $11.2 \mathrm{Nm}$. Compared with the single MRF braking, the combined braking torque increases by $72.3 \%$. The combined braking with the SMA and MRF can effectively overcome degradation of the performance of the MRF at high temperatures and ensure stable braking. This paper provides a theoretical basis for the design and manufacturing of MRF and SMA combined braking devices.

\section{Acknowledgments}

This research was supported by the National Natural Science Foundation of China (51875068) and the National Natural Science Foundation of China (51905060).

\section{References}

1. Aguiar R.A.A., Savi M.A., Pacheco P.M.C.L., 2010, Experimental and numerical investigations of shape memory alloy helical springs, Smart Materials and Structures, 19, 025008

2. An S., Ryu J., Chо M., Chо K., 2012, Engineering design framework for a shape memory alloy coil spring actuator using a static two-state model, Smart Materials and Structures, 21, 55009 
3. De V.J., Klingenberg D.J., Hidalgo-Alvarez R., 2011, Magnetorheological fluids: a review, Soft Matter, 7, 3701

4. Huang J., Zhang J.Q., Yang Y., Wei Y.Q., 2002, Analysis and design of a cylindrical magnetorheological fluid brake, Journal of Materials Processing Technology, 129, 559-562

5. Hwang Y.H., Kang S.R., Cha S.W., Choi S.B., 2019, A robot-assisted cutting surgery of human-like tissues using a haptic master operated by magnetorheological clutches and brakes, Smart Materials and Structures, 28, 065016

6. JАсов R., 1951, Magnetic Fluid Torque and Force Transmitting Device, United States Patent 2575360

7. Kim H.Y., Ikehara Y., Kim J.I., Hosoda H., Miyazaki S., 2006, Martensitic transformation, shape memory effect and superelasticity of Ti-Nb binary alloys, Acta Materialia, 54, 2419-2429

8. Le-Duc T., Ho-Huu V., Nguren-Quoc H., 2018, Multi-objective optimal design of magnetorheological brakes for motorcycling application considering thermal effect in working process, Smart Materials and Structures, 27, 75060

9. Li W.H., Du H., 2003, Design and experimental evaluation of a magnetorheological brake, International Journal of Advanced Manufacturing Technology, 21, 508-515

10. Ma J., Huang H., Huang J., 2013, Characteristics analysis and testing of SMA spring actuator, Advances in Materials Science and Engineering, 2013, 1-7

11. Ma J., 2013, Research on Magnetorheological Fluid Transmission and Application Driven by Shape Memory Alloy, Chongqing University

12. Mirzaeifar R., DesRoches R., Yavari A., 2011, A combined analytical, numerical, and experimental study of shape-memory-alloy helical springs, International Journal of Solids and Structures, 48, 611-624

13. Mohd J.J., Leary M., Subic A., Gibson M.A., 2014, A review of shape memory alloy research, applications and opportunities, Materials and Design, 56, 1078-1113

14. Muthalif A.G.A., Kasemi H.B., Nordin N.H.D., Rashid M.M., Razali M.K.M., 2017, Semi-active vibration control using experimental model of magnetorheological damper with adaptive F-PID controller, Smart Structures and Systems, 20, 85-97

15. Otsuka K., Ren X., 2005, Physical metallurgy of Ti-Ni-based shape memory alloys, Progress in Materials Science, 50, 511-678

16. Rabinow J., 1948, The magnetic fluid clutch, Transactions of the American Institute of Electrical Engineers, 67, 1308-1315

17. Sun T., Peng X., Li J., Feng C., 2013, Testing device and experimental investigation to influencing factors of magnetorheological fluid, International Journal of Applied Electromagnetics and Mechanics, 43, 283-292

18. Wang D.M., Hou Y.F., Tian Z.Z., 2013, A novel high-torque magnetorheological brake with a water cooling method for heat dissipation, Smart Materials and Structures, 22, 025019

19. WANG H., Bi C., 2020, Study of a magnetorheological brake under compression-shear mode, Smart Materials and Structures, 29, 17001 